Il reste encore à dire quelques mots de la conformation de l'extrémité abàominale. Je n'ai malheureusement pas pu étudier de mâle de Cylindrachaeta, les deux exemplaires en ma possession étant des femelles adultes, mais j'ai tout lieu de supposer que M. le Pr GiglioTos a interverti les sexes dans son étude précitée. Il indique en effet que le mâle aurait 7 sternites abdominaux et la femelle $8\left(^{1}\right)$; ce caractère serait tout à fait l'inverse de ce que l'on constate chez tous les Orthoptères; d'autre part, l'un des deux sexes - la femelle d'après lui - montre des valves anales inférieures denticulées; or, ce caractère est fréquent chez les Orthoptères et s'observe généralement chez les mâles. En fait, les deux individus que j'ai étudiés sont bien des femelles montrant 7 sternites abdominaux et la plaque sous-génitale $\left({ }^{2}\right)$; de même que chez les Tridactylus et la plupart des Curtillinae, il n'existe aucune trace d'oviscapte et le $9^{\text {e }}$ sternite est bien développé, dépassant la plaque sous-génitale; le $8^{\mathrm{e}}$ sternite est nul comme chez les Curtillinae. Les valves anales sont fortement sclérifiées, mais ne présentent aucun caractère exceptionnel; on peut seulement noter l'existence, entre la base des cerques et le $10^{\text {e }}$ tergite, d'une petite plaque triangulaire, qui semble représenter le $11^{\mathrm{e}}$ tergite.

\title{
Nephila Riverai, nouvelle Araignée argentine
}

\author{
par Jean Brèthes.
}

M. Joseph-Albert Rivera, estanciero à Curuzú-Cuatiá (province de Corrientes) à environ วัวั0 kilom. au nord de Buénos-Aires, vient de me communiquer une Araignée dont la soie, dit-il, pourrait peut-être avoir des applications industrielles. Au moment de cette communication, il Iui a été impossible de me procurer la soie en question, mais il m'a promis, à l'époque propice, de me faire part de ses recherches.

En attendant, je ferai connaître cette Araignée qui me parait distincte de toutes celles du genre connues jusqu'aujourd'hui.

Nephila Riverai, n. sp. - $q$ Chélicères et crochets noirs, lui-

(1) Il faut compter 8 et 9, Giglio-Tos ne tenant pas compte du $1^{\text {er }}$ sternite qui est presque fusionné avec le métasternum.

(2) De même que chez tous les Gryllides, la plaque sous-génitale de la femelle doit àvoir ici une origine intersegmentaire. Voir à ce sujet : L. СноPARD, Note préliminaire sur la conformation de l'extrémité abdominale des Orthoptères (Arch. Zool. exp. [1917]). 
sants; céphalothorax noir en dessus, mais couvert d'un fin velouté argenté, les bords bruns; abdomen de couleur terre de Sienne obscur, avec en dessus une tache basale jaune suivie de quatre ou cinq paires de petites taches de même couleur en disposition longitudinale et des dessins anastomosés dendritiques de la même couleur de chaque côté qui se dirigent soit en dessus, soit en dessous de l'abdomen. Pièce labiale et lames maxillaires d'un brun foncé tournant à l'orangé à l'extrémité. Sternum orangé, avec trois régions médianes plus ou moins contiguës noirâtres. Abdomen comme en dessus avec trois lignes transverses jaunes plus prononcées : une avant et une autre après le pli épigastrique et la troisième avant le groupe mamillaire. Les pattesmàchoires sont d'un blanc jaunâtre à la base, les trois derniers articles noirs. Les hanches et trochanters sont d'un jaune orangé en dessous, noirâtres sur le côté; les fémurs sont orangés et noirâtres vers l'extrémité. Les patellas sont d'un orangé plus ou moins obscur; les tibias sont orangés à la base et à l'extrémité avec un anneau subapical noirâtre; les protarses et tarses sont noirs avec la base de ceux-la orangée. Les épines des pattes sont toutes noires ainsi que les poils; au sternum et aux hanches, il y a de fins poils dorés.

La ligne antérieure des yeux est récurvée, la postérieure droite. Les quatre yeux médians forment un carré parfait et sont égaux entre eux, le groupe latéral est sur une légère élévation, les yeux étant espacés du double de leur diamètre et étant plus petits que les médians. De la fossette postérieure rayonnent de légères impressions; point de tubercules ni d'éminences. Le bandeau est d'une hauteur égale à la distance qui sépare les yeux médians. Les chélicères avec poils noirs épars; une petite impression vers la base des crochets, trois dents à peu près égales au côté supérieur et quatre au còté inférieur de la gouttière. Lames maxillaires arrondies au bord externe apical; pièce labiale en demi-ellipse. Troisième et sixième articles des pattes-mâchoires égaux en longueur, le cinquième un peu plus de la moitié du sixième, le quatrième égal à la moitié du cinquième.

Sternum cordiforme allongé, légèrement voûté, très peu relevé au milieu antérieur, en face des articulations des pattes I et II et à l'extrémité postérieure. Longueur relative des pattes : I, II, IV et III. Les pattes I un peu plus de deux fois aussi longues que III. Des touffes de poils noirs aux tibias 1, 2 et 4 , ces poils aussi longs que le diamètre du tibia; aux fémurs I et II, il y a aussi des poils noirs, mais bien plus courts et ne formant pas de touffes.

Abdomen oviforme, les bords antérieur et postérieur arrondis, les bords latéraux présentant la plus grande largeur vers le tiers anté- 
rieur. La fente de l'épigyne transverse, avec une très légère striation transverse au milieu.

Mesures : céphalothorax : $8 \times \ddot{\mathrm{mm}}$; abdomen vu d'en haut : $13 \times 6.3 \mathrm{~mm}$. Tout le corps $10 \ddot{\mathrm{mm}}$; pattes : I, 40 ; II, 31 ; III, 18 IV, $28 \mathrm{~mm}$.

Mâle inconnu.

République Argentine : province de Corrientes.

\section{Bulletin bibliographique.}

Blanchard (R.) : Éloge des zoologistes amateurs. (Bull. Soc. zool. Fr., XXIX [1914] p. 73-77).*

ID. : Charlatans et pseudo-parasites. Les "Vers des yeux ». (Bull. Soc. Path. exot., X [1918], p. כ̈79-ə̈8ə̈), fig.*

ID. : Larves de Névroptères éventuellement hématophages. (l. c., p. 586592), fig.*

Bolivar (C.) : Estudio monográfico de la sección "Trauliae ", Orth., Locustinae. (Rev. r. Acad. Cienc. exact., fis. y nat. Madrid, XV [1917], p. 60ڤ̈-644), fig.*

Boullet (E.) et Le Cerf (F.) : Catalogue de la collection de Lépidoptères du Muséum national d'Histoire naturelle de Paris. I, Papilionidae. 52 p. - Offert par M. le $\mathrm{D}^{\mathrm{r}} \mathrm{R}$. Blanchard.

Bouvier (E.-L.) : La vie psychique des Insectes. Paris, 1918, 300 p.* Buysson (H. DU) : Contributions à la faune du Maroc: Élatérides.

(Bull. Soc. Hist. nat. Afr. N., IX [1918], p. 109-112*

Cros (A.) : Le Meloë foveolatus Guérin. (Bull. Soc. Hist. nat. Afr. N., [1918], p. 38-104), fig.*

Demachy (E.) : Notes d'entomologie scientifique. - I. La localisation du sens de l'odorat chez les Insectes. - II. Le problème de l'Abeille. Origine et formation des neutres. - III. Le problème de l'Abeille. La théorie de Dzierzon. - Amélie-les-Bains, juin-juillet 1918*

Desbordes (H.) : Synonymie du Saprinus (Pachylopus) Chobauti Desb.

(Bull. Soc. ent. Fr. [1918], p. 15̈7-15̈8).*

Dodero (A.) : Coléoptères endogés de la Catalogne. Première note.

(Bull. Instit. catal. Hist. nat. [1918], p. 103-110).* 


\section{$2 \mathrm{BHL}$ Biodiversity Heritage Library}

Brèthes, Jean. 1918. "Nephila Riverai, nouvelle Araignee argentine." Bulletin de la Société entomologique de France 1918, 216-218.

View This Item Online: https://www.biodiversitylibrary.org/item/35447

Permalink: https://www.biodiversitylibrary.org/partpdf/144392

\section{Holding Institution}

Smithsonian Libraries

\section{Sponsored by}

Smithsonian

\section{Copyright \& Reuse}

Copyright Status: NOT_IN_COPYRIGHT

This document was created from content at the Biodiversity Heritage Library, the world's largest open access digital library for biodiversity literature and archives. Visit BHL at https://www.biodiversitylibrary.org. 